\title{
The emergence of interdisciplinary research in cancer diagnostics
}

\begin{abstract}
Cancer has been one of the leading causes of death globally for last few decades. Recently, it moved to the second position of this morbid chart. It was responsible for an astonishing 8.8 million deaths in 2015 claiming nearly $16 \%$ of all deaths according to a report published in 2017 by WHO. Due to its multi-faceted and not-so-well-understood origin, it has long been an elusive target for scientists and researchers around the globe. Even though cancer detection in its own right is an extremely crucial step for the treatment, but time is of essence here i.e. when it's detected is very important as it dictates the scope of the treatment. Detecting cancer at an earlier stage always gives an extra advantage providing more options for finding better treatment. Reasonably so, it is of paramount importance to have early detection capability of any modern point-of-care cancer diagnostic system. Despite the very biological nature of cancer, it is now widely believed that the solution to bring a good detection tool may not lie within the traditional boundary of biology. As a result, in last few decades, there's a tremendous efforts made around the globe to attempt the problem from a wide variety of perspectives. Due to this fact, a large number of interdisciplinary works has been initiated or done in past decade. In this brief review, the current status and recent past of interdisciplinary research in the field of cancer diagnostics is accounted, and the path of future cancer diagnostics will also be touched.
\end{abstract}

Keywords: Cancer, Early detection ,Biomarker, Aptamer, Biosensor, Diagnostics, Microfluidics, Nanostructure, Functionalization, Interdisciplinary research
Volume 6 Issue 3 - 2017

\section{Md Motasim Bellah}

Department of Electrical Engineering, University of Texas at Arlington, USA

Correspondence: Md Motasim Bellah, Department of Electrical Engineering, University of Texas at Arlington, Arlington, Texas 76019, USA Email bellah72@gmail.com

Received: October 29, 2017 | Published: November 28, 2017

\section{Introduction}

Cancer is the result of uncontrolled or poorly controlled proliferation of cells in an organism. It can be caused by wide varieties of reasons. The most prevalent causes of cancer are genetic mutation, inability to repair genes, and abnormal expression. These causes can be inherited or acquired or both over the life span of a host. For any disease to treat, the very first step is to detect its symptoms with reasonable confidence. Unfortunately, many cancer types go unnoticed for years until the host show noticeable symptoms and that makes it one of the most difficult physical conditions to detect. Due to this fact, cancer is often detected at the very later stage when the patients have very little or in many cases no options for treatment. That's why it is extremely important to be able to detect cancer at an earlier stage. It can greatly increase the chances for successful treatment. Two major components of early detection of cancer play a pivotal role in cancer treatment- public awareness to promote early diagnosis and clinical screening. There are few ways that can lead to early diagnosis such as recognizing warning signs and taking prompt action. If the awareness of possible warning signs of cancer among the general public along with physicians, nurses and other healthcare can be increased, then it can have a great impact on the diagnosis and treatment of the disease. Lumps, sores that fail to heal, abnormal bleeding, persistent indigestion, and chronic hoarseness are some early signs of cancer. Cancer screening involves the use of simple tests in order to identify individuals who have it, but do not have symptoms yet.

The dictionary definition of a sensor is a device that detects or measures a physical quantity and records, indicates or otherwise responds to it. From broader perspective, biosensor or transducer is a device that converts the interaction between an analyte and an active element into a measurable signal. When tumor cells start over or under expressing any protein or other molecules, biosensors are the right tool to detect them at an early stage. The first biosensor dates back to
1962 when Professor Leland C. Clark explained the mechanism of an oxygen probe and how it can be used to detect a range of analytes that could be measured with it. At a Symposium in the New York Academy of Sciences, he described how to make a glucose sensor using the already developed oxygen sensor.$^{1}$ Since then, biosensors are being developed and used for a numerous molecules and biomarkersattributes that have strong correlation to the inception of a disease. Due to the multitudes of possibilities of biosensor, cancer researchers from various parts of the world quickly adopted this new method in cancer diagnostics. The primary steps involved in any biosensing are recognition of analyte, signal transduction, and readout. Biosensors offer exciting opportunities for numerous decentralized clinical applications due to their specificity, speed, portability, and low cost.

\section{Interdisciplinary work in cancer diagnosis}

Since cancer can be caused due to a wide varieties of reasons, often its origin is not very well-understood. Ever since the very first malignant cells were detected from sputum in 1851 , there has been a plethora of devices and techniques developed to detect cancer at early stage. Brief history of cancer diagnosis is shown in Figure 1. It covers the major breakthroughs that changed the course of cancer diagnosis and treatment over century.

Out of varieties of detection method, the most promising candidate is probably biosensor. It has entered the stage of cancer diagnosis due to the versatility of their usages, high sensitivity, and high specificity ${ }^{2,3}$ Based on signal transduction methods i.e. the process of translating the interaction between the analyte with the detection element, biosensors may be classified into six basic groups- optical, electrochemical, biomechanical, mass, magnetic, and thermal sensors ${ }^{4}$ Most biosensors will fall either one of these categories or any combination of them. The fundamental mechanism of these biosensors are not limited in the domain of biology. Obviously all of them require 
advanced knowledge of biology, but it doesn't suffice to develop a biosensor without some interdisciplinary knowledge from electrical, chemical, or mechanical engineering. The part of the detection where engineering comes into play is the signal transduction. In the following sections, few of these interdisciplinary approaches of cancer detection will be briefly discussed.

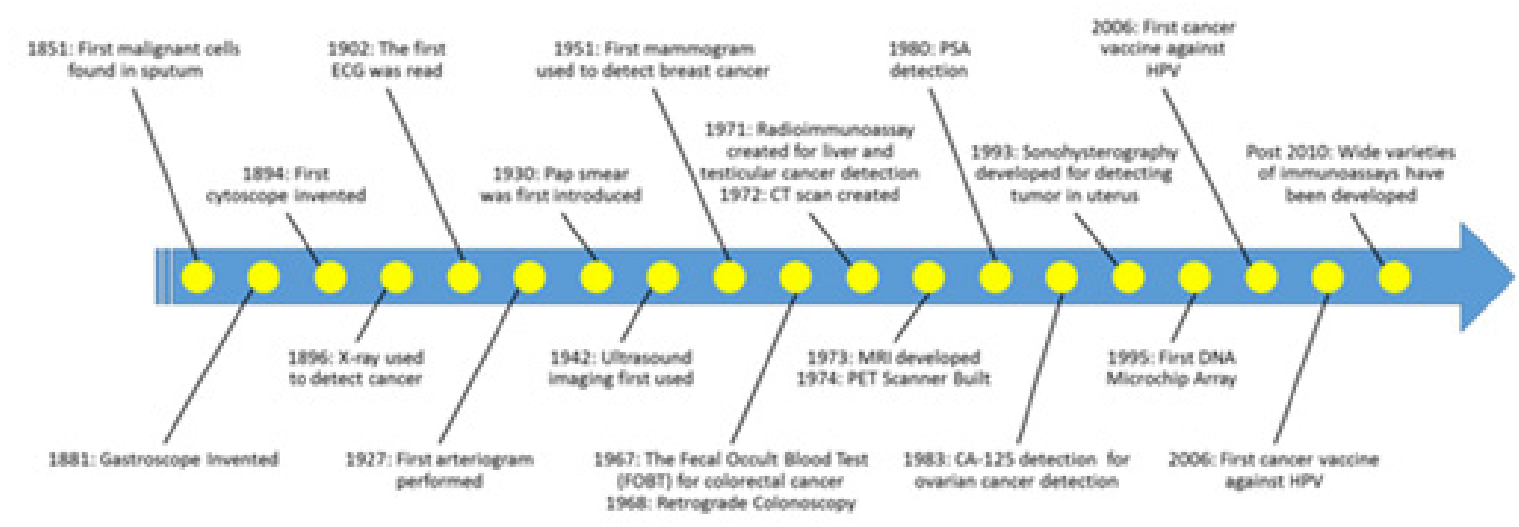

Figure I History of cancer diagnosis. ${ }^{5}$

\section{Magnetic and optical}

Prostate-specific antigen (PSA) was possibly the first tumor biomarker that was identified and put into routine clinical use for screening and diagnosis of prostate cancer..$^{5}$ The diagnosis was done through microscope using the morphological differences between cancer and healthy cells. Engineered surface with selective ligand can be used to capture cancer cells and detect them using optical or fluorescent microscope.$^{6}$ Another major optical biosensing mechanism is surface plasma resonance (SPR). Špringer et al. ${ }^{7}$ reported a method for the detection of colorectal cancer specific antigen (CEA) using bio-functionalized gold nanoparticles ${ }^{7}$ A similar approach was adopted by Joshi et al. ${ }^{8}$ to detect microRNA for pancreatic cancer detection. A femtomolar detection sensitivity was achieved exploiting the surface plasma resonance on the surface of gold nanoprisms . Other nanostructures may also be used as the transduction medium .${ }^{9}$ Optical waveguide is another detection tool that has attained some attentions lately.$^{10,11}$ Magneto-optical approach has been used to detect cancer biomarkers.$^{12}$ Not so prevalent pure magnetic approach has also been reported to detect breast cancer at early stage.${ }^{13}$ But in most cases, magnetic approach is usually complemented with another method to enhance detectability.

\section{Electrochemical}

Traditionally, electrochemical biosensors occupied the major share of the biosensors development due to their greater potential to provide simple, inexpensive and yet accurate and sensitive platform for disease diagnosis.$^{2,14}$ A number of promising electrochemical strategies have been performed for cancer biomarkers detection including voltammetric techniques, amperometric, and impedimetric. In a voltammetric setting, two or three electrode electrochemical cell systems together with a potentiostat are used to sense the transduction signal. This method is reportedly used to detect quite a few cancer biomarker with great sensitivity and specificity ${ }^{15-18}$ In an amperometric technique, the transduction happens through current measurement. Usually the performance of an amperometric sensor is heavily dependent on the electrode properties. The sensitivity of this type of sensor can go down to $\mathrm{fM}$ concentration of the analytes.$^{20-23}$

\section{Biomechanical}

A change in the mechanical properties of cells is often entailed by alterations in biological activities and transformation of cell states.
Recently, cell stiffness/elasticity has been emerged as a marker for cellular phenotypic events and diseases. Lower stiffness is commonly reported in the malignant cancer cells than normal cells.$^{23-26}$ Cytoskeleton component, especially actin microstructure primarily determines the mechanical properties of a cell . 27-29 Out of several biomechanical approaches, micropipette aspiration, optical stretcher and atomic force microscopy are the main few that are widely used in cancer detection. The underlying principle is to apply mechanical force onto cell and measure the response to determine the exoskeleton stiffness. Usually some variants of modulus of elasticity are computed to compare cancer cells and healthy cells.

\section{Mass and thermal}

One of the earlier approaches to detect cancer is to use the mass of the biomarker. The surface of a piezoelectric resonator or a cantilever works as the active surface. The target is selectively attached to the resonator using a ligand (antibody or aptamer). The sensing signal is measured in terms of the resonance frequency of the piezoelectric crystal.$^{30,31}$ Thermal technique is probably the least sought approach in cancer detection. In many modalities, heat may be involved but rarely used as the actual transduction signal for the detection. However, there has been few studies that reportedly used the properties of heat transfer as the signal. Eersels et al. ${ }^{33}$ studied the binding of cancer cell and functionalized surface using the heat transfer rate ${ }^{33}$ In recent studies, it has been found that stress hormones such as noradrenaline are involved in the initiation and progression of tumors.$^{33,34} \mathrm{~A}$ simple heat transfer method with synthetic receptors can be used to develop biosensor that is capable of detecting neoadrenaline like biomarkers 35

\section{Conclusion}

Even after decades of cutting-edge research and investigation, cancer is still far from being a solved case. Synergistic efforts are continuously being made to bring knowledge from seemingly unrelated fields in order to develop novel detection method for cancers. So far, there has been tremendous progress made in the area of increasing awareness as well as the routine screening methods along with more and more efficient and effective diagnosis techniques, which directly impacts the life expectancy of cancer patients. Due to these newly adopted preventive and diagnostic measures, $70 \%$ of all children cancer patients are now cured. The life expectancy of cancer 
patient has been extended quite significantly over the last decade. Two out of three people in the United States are now living more than five years after their diagnosis ${ }^{36,37}$ The response rates to treatments across all cancer types have nearly doubled within the last decade alone. Despite the medical breakthroughs we have made so far, cancer is still claiming scores of lives annually across the globe. As we have already learned though large number of methods and approaches that we have tried so far that interdisciplinary research in cancer diagnosis holds great promise for the future of this field, it underscores the importance of continuing the stride in the same direction. Still there are many areas that are unexplored and waiting to be discovered. From the observed trend in the last few decades, it can be inferred that the scenario of cancer diagnosis will continue to be reshaped as we keep adopting more collaborative and holistic approach to tackle it. More interdisciplinary research and development will inevitably emerge out of this effort to resolve this century old health problem. The future of cancer diagnosis and treatment will largely be determined by how well we will be able to apply our synergistic expertise from multiple discipline.

\section{Acknowledgements}

None.

\section{Conflicts of Interest}

None.

\section{References}

1. Mascini M, Palchetti I Biosensor Technology: A Brief History. Sensors and Microsystems. 2010;404:2869-2875.

2. Turner, Anthony, George Wilson, Isao Kaube Biosensors: Fundamentals and Applications. Oxford University Press, UK, pp. 1987;770.

3. Banica Florinel-Gabriel Chemical Sensors and Biosensors: Fundamentals and Applications. John Wiley \& Sons, UK, pp. 2012;576.

4. Ivnitski, Dmitri, Ihab Abdel-Hamid et al. Biosensors for detection of pathogenic bacteria. Biosensors and Bioelectronics. 1999;14 (7):599-624.

5. Smith DS, Humphrey PA, Catalona WJ The early detection of prostate carcinoma with prostate specific antigen: the Washington University experience. Cancer. 1997;80(9):1852-1856.

6. Islam M, Bellah MM, Sajid A et al. Effects of Nanotexture on Electrical Profiling of Single Tumor Cell and Detection of Cancer from Blood in Microfluidic Channels. Scientific Reports. 2015;5(1):13031.

7. Špringer T, Homola J Biofunctionalized gold nanoparticles for SPRbiosensor-based detection of CEA in blood plasma. Analytical and Bioanalytical Chemistry. 2012;404(10):2869-2875.

8. Joshi GK, Deitz-McElyea S, Johnson M et al. Highly Specific Plasmonic Biosensors for Ultrasensitive MicroRNA Detection in Plasma from Pancreatic Cancer Patients. Nano letters. 2014;14(12):6955.

9. Motasim Bellah, Shawn M Christensen, Samir M Iqbal Nanostructures for Medical Diagnostics. Journal of Nanomaterials. 2012

10. Harshini Mukundan, Jessica Z Kubicek, Alicia Holt et al. Planar optical waveguide-based biosensor for the quantitative detection of tumor markers Sensors \& Actuators: B. Chemical. 2009;138(2):453-460.

11. Taeb A, S Gigoyan, Safavi-Naeini Millimetre-wave waveguide reflectometers for early detection of skin cancer. IET Microwaves, Antennas \& Propagation. 2013;7(14):1182-1186.

12. Hu Y, Li L, Guo L The sandwich-type aptasensor based on gold nanoparticles/DNA/magnetic beads for detection of cancer biomarker protein AGR2. Sensors and Actuators B: Chemical. 2015;209:846-852.
13. Hathaway HJ, Butler KS, Adolphi NL et al. Detection of breast cancer cells using targeted magnetic nanoparticles and ultra-sensitive magnetic field sensors. Breast cancer research: BCR. 2011;13(5):108R.

14. Wang J, Kawde A Pencil-based renewable biosensor for label-free electrochemical detection of DNA hybridization. Anal Chim Acta. 2000;431:219-225.

15. Zhao C, Lin D, Wu J et al. Nanogold-Enriched Carbon Nanohorn Label for Sensitive Electrochemical Detection of Biomarker on a Disposable Immunosensor. Electroanalysis. 2013;25(4):1044-1049.

16. Duangkaew P, Tapaneeyakorn S, Apiwat C et al. Ultrasensitive electrochemical immunosensor based on dual signal amplification process for p16(INK4a) cervical cancer detection in clinical samples. Biosensors \& bioelectronics. 2015;74:673.

17. Erdem A, E Eksin, Congur Indicator-free electrochemical biosensor for microRNA detection based on carbon nanofibers modified screen printed electrodes. Journal of Electroanalytical Chemistry. 2015;755:167-173.

18. Kong FY, Xu MT, Xu JJ et al. A novel lable-free electrochemical immunosensor for carcinoembryonic antigen based on gold nanoparticles-thionine-reduced graphene oxide nanocomposite film modified glassy carbon electrode. Talanta. 2011;85(5):2620-2625.

19. Bellah MM, SM Iqbal, Y Kim Differential behavior of EGFR-over expressing cancer cells through aptamer-functionalized micropores. Microfluidics and Nanofluidics. 2014;17(6):983-992.

20. Islam M, Sajid A, Mahmood MA et al. Nanotextured polymer substrates show enhanced cancer cell isolation and cell culture. Nanotechnology. 2015;26(22):225101.

21. Azzouzi S, Rotariu L, Benito AM et al. A novel amperometric biosensor based on gold nanoparticles anchored on reduced graphene oxide for sensitive detection of 1-lactate tumor biomarker. Biosens Bioelectron. 2015;69:280-286.

22. Zhang Q, Chen X, Tang Y, Ge L, Guo B Amperometric carbohydrate antigen 19-9 immunosensor based on three dimensional ordered macroporous magnetic $\mathrm{Au}$ film coupling direct electrochemistry of horseradish peroxidase. Analytica Chimica Acta. 2014;815:42-50.

23. Torrente-Rodríguez RM, S Campuzano, E López-Hernández et al. Direct Determination of miR-21 in Total RNA Extracted from Breast Cancer Samples Using Magnetosensing Platforms and the p19 Viral Protein as Detector Bioreceptor. Electroanalysis. 2014;26(10):2080-2087.

24. Suresh S, Spatz J, Mills JP et al. Connections between single-cell biomechanics and human disease states: gastrointestinal cancer and malaria. Acta Biomaterialia. 2005;1(1):15-30.

25. Guck J, Schinkinger S, Lincoln B et al. Optical deformability as an inherent cell marker for testing malignant transformation and metastatic competence. Biophys J. 2005;88(5):3689-3698.

26. Rao J, Jin Y, Gimzewski JK, Cross SE Nanomechanical analysis of cells from cancer patients." Nature Nanotechnology. 2007;2(12):780-783.

27. Dai J, MP Sheetz Mechanical properties of neuronal growth cone membranes studied by tether formation with laser optical tweezers. Biophysical Journal. 1995;68(3):988-996.

28. Trickey WR, Vail TP, Guilak F The role of the cytoskeleton in the viscoelastic properties of human articular chondrocytes. Journal of Orthopaedic Research. 2004;22(1):131-139.

29. Guck J, R Ananthakrishnan, TJ Moon Optical deformability of soft biological dielectrics. Physical Review Letters. 2000;84(23):5451-5454.

30. Zhang K, Zhao L, Zhao X et al. A microfluidic system with surface modified piezoelectric sensor for trapping and detection of cancer cells." Biosensors and Bioelectronics. 2010;26(2):935-939.

31. Su L, L Zou, C Fong et al. Detection of cancer biomarkers by piezoelectric biosensor using PZT ceramic resonator as the transducer. Biosensors \& Bioelectronics. 2013;46:155. 
32. Eersels K, B van Grinsven, T Vandenryt et al. Improving the sensitivity of the heat-transfer method (HTM) for cancer cell detection with optimized sensor chips. Physica Status Solidi A-applications and Materials Science. 2015;212(6):1320-1326.

33. Sood AK, Bhatty R, Kamat AA et al. Stress hormone-mediated invasion of ovarian cancer cells. Clinical Cancer Research. 2006;12(2):369-375.

34. Bast RC Jr, Hennessy B, Mills GB The biology of ovarian cancer: new opportunities for translation." Nature Reviews Cancer. 2009;9(6):415-428.
35. Choi MJ, Cho KH, Lee $\mathrm{S}$ et al. hTERT mediates norepinephrineinduced Slug expression and ovarian cancer aggressiveness. Oncogene. 2015;34(26):3402.

36. S Jane Henley, Simple D Singh, Jessica King, Reda Wilson, Mary Elizabeth O'Neil, et al. Invasive Cancer Incidence and Survival United States, 2011. Morbidity and Mortality Weekly Report (MMWR). 2011;64(9): 237-242.

37. Cancer Quest: History of Cancer Detection 1851-1995. 